\title{
1 Oxicam-type NSAIDs enhance Agrobacterium-mediated 2 transformation in plants
}

3

4

5

6

7

8

9

Seung-won Choi ${ }^{1,2}$, Kie Kumaishi ${ }^{3}$, Reiko Motohashi ${ }^{4}$, Harumi Enoki ${ }^{4}$, Wiluk Chacuttayapong ${ }^{4}$, Tadashi Takamizo ${ }^{5}$, Hiroaki Saika6, Masaki Endo ${ }^{6}$, Tetsuya Yamada7, Aya Hirose7, Nobuya Koizuka $^{8}$, Seisuke Kimura ${ }^{9}, 10$, Yaichi Kawakatsu' ${ }^{9}$, Hiroyuki Koga11, Emi Ito², Ken Shirasu1,*, Yasunori Ichihashi ${ }^{3, *}$

${ }^{1}$ Riken Center for Sustainable Resource Science, Yokohama, Kanagawa, Japan.

2 Department of Natural Sciences, International Christian University (ICU), Osawa 3-10-2, Mitaka, Tokyo 181-8585, Japan.

${ }^{3}$ Riken BioResource Research Center, Tsukuba, Ibaraki 305-0074, Japan.

${ }^{4}$ Faculty of Agriculture, Department of Applied Life Sciences, Shizuoka University, 836 Ohoya Suruga-ku, Shizuoka, Shizuoka 422-8529, Japan.

${ }^{5}$ National Institute of Livestock and Grassland Science, Nasushiobara, Tochigi 329-2793, Japan

6 Institute of Agrobiological Sciences, National Agriculture and Food Research Organization (NARO), Tsukuba, Ibaraki 305-8634, Japan.

${ }^{7}$ Graduate School of Agriculture, Hokkaido University, Kita9 Nishi9, Kita-ku, Sapporo, Hokkaido 060-8589, Japan.

8 College of Agriculture, Tamagawa University, 6-1-1 Tamagawagakuen, Machida, 194-8610, Japan

${ }^{9}$ Department of Industrial Life Sciences, Faculty of Life Sciences, Kyoto Sangyo University, Kitaku, Kyoto 603-8555, Japan

${ }^{10}$ Center for Plant Sciences, Kyoto Sangyo University, Kamigamo-motoyama, Kita-ku, Kyoto 603-8555, Japan

11 Department of Biological Sciences, Graduate school of Sciences, The University of Tokyo, Bunkyo, Tokyo 113-0033, Japan

* Correspondence:

Yasunori Ichihashi, yasuhnori.ichihashi@riken.jp; Ken Shirasu, ken.shirasu@riken.jp

\begin{abstract}
Agrobacterium-mediated transformation represents a key innovation for plant breeding and is routinely used in research and applied biology. However, for several species, the efficacy of transformation is limited. In this study, we discovered that oxicam-type nonsteroidal antiinflammatory drugs (NSAIDs), including tenoxicam (TNX), enhance the efficiency of Agrobacterium-mediated transient transformation in the model species Arabidopsis thaliana via leaf infiltration and can be successfully applied in analyses of the subcellular localisation of fluorescent fusion proteins. TNX acts as an inhibitor of plant immune responses and lacks similar transient transformation efficiency in a dde2/ein2/pad4/sid2 quadruple mutant background, thereby indicating that T NX increases the efficiency of Agrobacterium infection via a transient shutdown of the immune system mediated by jasmonic acid, ethylene, and salicylic acid signalling. In addition, we found that TNX enhances the efficiency of stable transformation in crops of agricultural and economic importance, such as Jatropha and maize, indicating that TNX can enhance the integration of exogenous DNA into the plant genome via an increased introduction of DNA into plant cells. Given that treatment with oxicam compounds is simple,
\end{abstract}


47 cost effective, and has broad utility, we anticipate that this discovery will contribute to

48 accelerating genome-editing technologies in plants.

49

50

\section{Keywords}

51 Agrobacterium tumefaciens, Arabidopsis thaliana, Jatropha curcas, oxicam, NSAIDs, Tenoxicam, 52 Genetic transformation, Zea mays

53

54

\section{Introduction}

The continual increase in global population poses considerable challenges with respect to maintaining the overall sustainability of food production, and in this regard, the genetic modification of crops via plant transformation is seen as providing one solution to cope with prospect of future food insecurity. Owing to advances in sequencing technology, whole-genome sequences of many plant species, including crops, have been determined, thereby enabling comprehensive genetic analyses (The Arabidopsis Genome Initiative, 2000; Goff et al., 2002; Yu et al., 2002). In addition, recent technical advances in genome editing, such as TAL effector nucleases and CRISPR/Cas, are facilitating the generation of targeted DNA double-strand breaks with high specificity in complex genomes. Therefore, technically, the manipulation of plant genomes via genetic transformation is becoming increasingly viable. However, in the case of many plant species, including crops of agricultural and economic importance, current modification approaches remain inefficient. Accordingly, in order to maximise the potential implementation of technological knowledge to feed the world, it is essential to further enhance the capacity and efficiency of plant transformation (Altpeter et al., 2016).

Common soil bacteria in the genus Agrobacterium have been found to have the unique ability of interkingdom genetic transfer, notably, the incorporation of transfer DNA (T-DNA) segments into plant genomes (Escobar and Dandekar, 2003; Tzfira et al., 2004). This ability of Agrobacterium species to transfer DNA to plants has been extensively exploited for genetic engineering purposes by replacing oncogenes within bacterial T-DNA with any gene of interest. Agrobacterium-mediated genetic transformation has become a routine procedure in basic plant research as well as a principal means of generating transgenic plants for the agricultural biotechnology industry (Gelvin, 2005). However, some plant species show heightened immune responses to suppress Agrobacterium-mediated transformation. For example, it is well established that ethylene (ET)- and salicylic acid (SA)-mediated immune responses are deployed by plants to restrict Agrobacterium-mediated transformation (Gaspar et al., 2004; Yuan et al., 2007; Anand et al., 2008; Nonaka et al., 2008b, 2008a; Lee et al., 2009). Indeed, immunity-related mutants and transformants have been reported to enhance the efficacy of Agrobacterium-mediated transient transformation [e.g. dde2/ein2/pad4/sid2 (Tsuda et al., 2009) and NahG (Rosas-Díaz et al., 2017)]. As an alternative strategy, transformation efficiency can also be enhanced by modifying the virulence of Agrobacterium, which influences plant immunity (Hiei et al., 1994; Ishida et al., 1996; Piers et al., 1996; Rashid et al., 1996; de Groot et al., 1998; Wenck et al., 1999; Rohini and Rao, 2000; Kunik et al., 2001; Komari et al., 2006; Kimura et al., 2015). Accordingly, controlling the immunity responses of target plants is considered a key strategy that could be used to address current inefficiencies in plant transformation.

During the course of previous chemical screening (Noutoshi et al., 2012), we established that oxicam-type nonsteroidal anti-inflammatory drugs (NSAIDs), such as tenoxicam (TNX), can function as plant immune inhibitors (Ishihama et al., 2020). In the present study, we report that brief treatment with immune-inhibiting oxicams is effective in enhancing transformation 
94 efficiency in several plant species, including agricultural crops. Although the effect of these

95

96

97

98

99

100

101

102

103

104

105

106

107

108

109

110

111

112

113

114

115

116

117

118

119

120

121

122

123

124

125

126

127

128

129

130

131

132

133

134

135

136

137

138

139

140

chemicals appears to be tissue specific, we believe that this simple approach, entailing the use a transient chemical treatment, has considerable potential to facilitate the practical application of technological principles for the purposes of plant transformation.

\section{Results and Discussion}

\section{Oxicam treatment enhances the efficiency of Agrobacterium-mediated transient transformation of Arabidopsis leaves}

The suppression of plant immune responses represents a key strategy for enhancing the efficiency of Agrobacterium-mediated transient transformation. In A. thaliana, agroinfiltration of leaves has been used to characterise gene functions in several mutants and transformants defective in immune responses (Tsuda et al., 2009; Sardesai et al., 2013; Rosas-Díaz et al., 2017). However, this approach would not be a suitable system for investigating the functions and subcellular localisation of unknown genes, as it does not exclude the possibility that such mutations may affect the functions of these genes. On the basis of the findings of our previous study, indicating that tenoxicam (TNX) inhibits plant immunity [Fig. 1; Ishihama et al. (2020)], we sought in the present study to determine whether a brief treatment of an Agrobacterium suspension with TNX would enable us to utilise wild-type $A$. thaliana for agroinfiltration in a manner similar to immunity-deficient mutants. To assess the feasibility of this approach, we added TNX to a suspension culture of 35S::GUS-carrying Agrobacterium prior to infiltration as a brief treatment, and compared the efficiency of transient transformation when the same culture without TNX treatment was used to agroinfiltrate the quadruple mutant (dde2/ein2/pad4/sid2), defective in ET-, SA-, and jasmonic acid (JA)-mediated immunity, which displays high efficiency (Tsuda et al., 2009). We accordingly observed that TNX-treated wild-type plants showed markedly enhanced GUS activity, which was seven times higher than that in plants transformed with non-treated Agrobacterium, and established that this heightened activity was equivalent to that observed when using non-treated Agrobacterium to infiltrate the mutant leaves (Fig. 2A and $B$ ). These findings thus provide evidence that the efficiency of DNA transformation was elevated owing to an increased efficiency in infection achieved by reducing plant immunity via a brief treatment with TNX. We subsequently examined the subcellular localisation of expressed marker proteins, including nuclear-localised histone 2B (H2B-GFP), the trans-Golgi network (TGN) marker Venus-SYP61, and plasma membrane-bound aquaporin (PIP2a-mCherry) following infiltration with TNX-treated Agrobacterium. We accordingly observed the anticipated localisation of these proteins in both TNX-treated and non-treated leaves (Fig. 2B), indicating that transient application of TNX can be effectively used to monitor the subcellular localisation of proteins. In contrast, however, TNX treatment appeared to have no similar effects with regards to root and flower transformation (Table S1 and S2). To determine the immune-related pathways targeted by TNX in recipient plants, we subjected the $d d e 2 / e i n 2 / p a d 4 / \operatorname{sid} 2$ quadruple mutant to transfection using TNX-treated Agrobacterium, and found that the efficiency of transient transformation, as indicated by GUS activity, in the quadruple mutant was similar to that in the mutant infiltrated with non-treated cells (Fig. 2C). These observations thus indicate that TNX can effectively inhibit the signalling pathways mediated by SA, JA, and ET. In addition to TNX, we also examined the effects of five other oxicams, namely, meloxicam (MLX), piroxicam (PRX), ampiroxicam (APRX), sudoxicam (SDX), and lornoxicam (LNX, Fig. 1A), on Agrobacterium-mediated transient transformation of Arabidopsis leaves, and accordingly observed that all five increased GUS activity. Indeed, LNX and SDX were found to be significantly 
141 effective, similar to TNX (Fig. 2D). These results thus indicate that transient oxicam treatment represents a highly effective new approach for enhancing the efficiency of leaf agroinfiltration in wild-type A thaliana.

\section{TNX can be applied for the transformation of agricultural crops}

On the basis of our finding that transient treatment with TNX can enhance the efficiency of Agrobacterium-mediated DNA transformation of the vegetative shoot tissues of $A$. thaliana (Fig. 2 ), we anticipated that similar treatment might also enable us to enhance the efficiency of transformation of the vegetative tissues of agricultural crops. To verify this possibility, we evaluated the effect of TNX on Agrobacterium-mediated transformation of Jatropha (J.curcas) and maize, as the transformation methods used for these species typically utilise vegetative tissues such as cotyledon explants and immature embryos, respectively [Fig. 3A-B, Fig. 4A; Ishida et al. (1996); Enoki et al. (2017)]. Given that Jatropha is an oil seed plant that yields biofuel, and that the seed oil can be readily processed as a replacement for petroleum-based diesel fuel (Fig. 2A, Forson et al., 2004), it would be desirable to develop techniques that can be used to enhance the transformation efficiency of Jatropha for industrial applications. In the present study, we performed Jatropha transformation by adding TNX to the co-cultivation suspension, and to determine the stability of transformation, we introduced selection vectors that enabled us to evaluate the transformation rate by PCR genotyping for the transgenes (Fig. 2C). We accordingly found that upon TNX treatment the rate of stable transformation with respect to the number of transformed soil-acclimated plants $(11.5 \%-18.8 \%)$ was approximately 4- to 16 -fold higher than that of the control plants (1.15\%-2.63\%, Fig. 3C). Similarly, when we calculated the transformation rate with respect to the number of cotyledon explants as starting materials, the rate was still 8-fold higher in the TNX treatment $(0.285 \%)$ than that in the control $(0.033 \%)$ for experiment 1 (Fig. 2 C). Collectively, these data indicate that pre-treatment with TNX can significantly enhance the efficiency of stable Agrobacterium-mediated transformation of Jatropha.

Maize is an important food in many countries and has a range of biotechnologicalrelated traits of commercial interest. Accordingly, maize is considered one of the most important target crops for the application of biotechnology (Que et al., 2014). In the present study, we performed transformation of maize using immature embryos (Fig. 4A) by adding TNX to the co-cultivation suspension or medium in six independent experiments using different maize strains. Using selection medium, we assessed the survival rate of callus derived from the TNX-treated and non-treated immature embryos, and in all independent experiments observed survival rates in the TNX treatments that were comparable to or higher than those obtained in the control treatments, particularly with respect to the Mi29 strain, which showed high sensitivity to TNX in terms of transformation efficiency (Fig. 4B). Surprisingly, we found that the TNX-treated calli tended to grow larger (Fig. 4C), thereby indicating that a larger number of cells within the immature embryos had been transformed in response to the TNX treatment to form a larger callus mass.

\section{Meristematic tissues are insensitive to tenoxicam}

We also evaluated the effect of T NX treatment on the efficiency of transformation for other plant species, namely rice, soybean, Brassica napus, Brassica rapa, and water starwort. Unfortunately, we failed to detect a significant effect of TNX treatment on the efficiency of transformation for these plants (Fig. S1, Table S3-S6). However, with the exception of water starwort, we used meristematic tissues of these plant, such as callus, callus-induced tissues, or cotyledonary nodes, rather than mature vegetative tissues, thereby indicating that the effect of TNX may be tissue dependent. In this context, it is well established that plant growth and immunity show antagonistic interactions; for example, young tissues must suppress the immune response to 
191

maximise growth in the absence of perceived pathogens, whereas mature organs are more adapted for defensive roles (Wang and Wang, 2014). This is supported by the different defencerelated responses observed during different phases of the cell cycle (Kadota et al., 2004). In this regard, SA has been established to be a key regulator promoting immunity but suppressing growth, whereas the atypical E2F protein DEL1 promotes cell proliferation and suppresses expression of the SA transporter gene enhanced disease susceptibility 5 (EDS5) and the SA biosynthetic gene ISOCHORISMATE SYNTHASE1 (ICS1) to suppress SA accumulation and defence responses in growing tissues (Chandran et al., 2014). Given that meristematic tissues already show less pronounced immune responses and that TNX can inhibit immune signalling pathways, including the SA pathway, we speculate that these meristematic tissues could be insensitive to TNX. This would be consistent with our observations that EDS5 and ICS1 are highly expressed in the mature tissues of $A$. thaliana, such as leaves, whereas expression is lower in meristematic tissues (Figure S2), which would explain why TNX, although efficient with respect to leaf agroinfiltration, appears to be ineffective for the transformation of flowers and roots (Fig. 2 and Tables S1 and S2).

\section{Conclusions}

In this study, we demonstrated that oxicam-type NSAIDs, including tenoxicam, can enhance the efficiency of Agrobacterium-mediated transformation in A. thaliana, as well as in certain crops of agricultural and economic importance, namely, Jatropha and maize. Given that treatment with oxicam compounds is comparatively straightforward (simply adding chemicals to the Agrobacterium co-cultivation medium), cost effective (estimate one US dollar for one hundred transformants), and has broad utility (it can readily be applied using existing methods), we anticipate that this discovery will contribute to research applications such as transient transformation assays, as well as potentially providing a solution for worldwide food security problems. However, we found that TNX had no appreciable effect with respect to the efficiency of meristematic tissue transformation, which we suspect might be attributable to the insensitivity of such tissues to TNX, given that the immune responses of meristematic tissues are typically suppressed. Therefore, we propose that future research in this area should focus on the monitoring and modification of plant immune responses, which we believe would provide a basis for overcoming some of the current limitations associated with plant transformation.

\section{Materials and Methods Arabidopsis thaliana}

For the purposes of agroinfiltration, we used 5- to 6-week-old Col-0 and dde2/ein2/pad4/sid2 quadruple mutant Arabidopsis plants grown in soil under short-day conditions (8/16 h light/dark). Agrobacterium tumefaciens (strain Agl1) carrying the GUS reporter gene in a pGreen 0029 vector used for infiltration was prepared and resuspended at an $O D_{600}$ of 0.2 , as previously described (Choi et al., 2013). For co-expression of organellar markers, $A$. tumefaciens (strain GV3101) transformants harbouring RPS::H2B-GFP, 35S::Venus-SYP61, and 35S::PIP2A$m$ Cherry were mixed after resuspension. Oxicams [tenoxicam (T0909; Sigma-Aldrich), meloxicam (M3935), piroxicam (P5654), ampiroxicam (SML1475), lornoxicam (SML0338), and sudoxicam (S688950; Wako Chemicals) dissolved in DMSO] were added to a final concentration of $100 \mu \mathrm{M}$ in the resuspension medium immediately prior to infiltration. Within $48 \mathrm{~h}$, GUS activity was measured using MUG assays and subcellular localisation was observed under a confocal microscope. 
For histochemical staining to visualise GUS activity, we used 5-bromo-4-chloro-3indolyl- $\beta$-D-glucuronide (X-gluc) as a substrate. Infiltrated leaves were collected and fixed in $90 \%$ acetone on ice for $15 \mathrm{~min}$, after which the samples were placed in GUS staining solution [1 $\mathrm{mg} / \mathrm{ml} \mathrm{X-gluc,} 100 \mathrm{mM} \mathrm{NaPO}_{4}\left(\mathrm{pH}\right.$ 7), $0.5 \mathrm{mM} \mathrm{K}_{3} \mathrm{Fe}(\mathrm{CN})_{6}, 0.5 \mathrm{mM} \mathrm{C}_{6} \mathrm{~N}_{6} \mathrm{FeK}_{4}, 10 \mathrm{mM}$ EDTA, and $0.1 \%$ triton $\mathrm{X}-100]$ under vacuum at room temperature for $15 \mathrm{~min}$ and incubated overnight at $37^{\circ} \mathrm{C}$. Subsequent to GUS detection, leaves were rinsed with $70 \%$ ethanol and cleared in chloral hydrate:water (2.5:1).

For quantitative MUG assays, leaf discs were homogenised in extraction buffer $(50 \mathrm{mM}$ $\mathrm{NaHPO}_{4} \mathrm{pH}$ 7.0, $10 \mathrm{mM}$ EDTA, 0.1\% sarcosyl, 0.1\% Triton X-100, and $10 \mathrm{mM} \beta$ -

mercaptoethanol) and centrifuged at $4^{\circ} \mathrm{C}$. Ten microlitres of the supernatant was added to $90 \mu \mathrm{L}$ of assay buffer [ $2 \mathrm{mM} 4$-methylumbelliferyl- $\beta$-D-glucuronic acid (MUG) in extraction buffer without $\beta$-mercaptoethanol] in wells of a 96 -well microplate and incubated at $37^{\circ} \mathrm{C}$ in the dark for $30 \mathrm{~min}$. Aliquots $(10 \mu \mathrm{L})$ of the reaction mixtures were mixed with $90 \mu \mathrm{L}$ of $\mathrm{Na}_{2} \mathrm{CO}_{3}$ and the fluorescence of 4-methylumbelliferone (4-MU) was measured at excitation at $365 \mathrm{~nm}$ and emission at $455 \mathrm{~nm}$ using a Mithras LB940 multiplate reader (Berthold Technologies). GUS activity was calculated as pmol 4-MU formed per mg total protein per min.

Subcellular localisation was observed using a Zeiss LSM 700 inverted confocal microscope. GFP and Venus were excited at $488 \mathrm{~nm}$, and emission was recorded between 501 and $545 \mathrm{~nm}$, whereas mRFP was excited at $561 \mathrm{~nm}$ and the emission was recorded between 570 and $615 \mathrm{~nm}$.

For root transformation, Col-0 plants were grown for more than 14 days on a plate containing Murashige and Skoog (MS) salt mixture (Wako Pure Chemical Industries, Ltd, Osaka, Japan) under long-day conditions (16/8 h light/dark). Roots were cut into 5-mm segments, and bundles of roots were inoculated with $A$. tumefaciens (GV3010) carrying 35S: GFP on an MS plate. Prior to application, DMSO or $100 \mu \mathrm{M}$ TNX was added to the Agrobacterium suspension. After 2 days of co-cultivation, transiently expressed GFP signals were observed from root bundle tips, and the number of tips expressing GFP was counted. The root bundles were then transferred to callus-inducing medium [CIM: $1 \times$ MS, $0.05 \mathrm{M}$ MES, vitamins $(0.5 \mathrm{mg} / \mathrm{L}$ nicotinic acid, $0.5 \mathrm{mg} / \mathrm{L}$ pyridoxine, $0.5 \mathrm{mg} / \mathrm{L}$ thiamine-HCl), $100 \mathrm{mg} / \mathrm{L}$ myo-inositol, $20 \mathrm{~g} / \mathrm{L}$ glucose, 5 $\mathrm{mg} / \mathrm{L}$ indole-3-acetic acid (IAA), $0.5 \mathrm{mg} / \mathrm{L}$ 2,4-dichlorophenoxy acetic acid (2,4-D), $0.3 \mathrm{mg} / \mathrm{L}$ kinetin, $\mathrm{pH}$ 5.7, and $7.5 \mathrm{~g} / \mathrm{L}$ Bacto agar]. After 4 weeks, callus had developed from all root bundles, and the number of GFP signal spots on callus. detected using a Leica M165 C stereomicroscope, was counted.

For floral-drop transformation, A. tumefaciens (strain Agl1) harbouring 35S::GUS was grown overnight at $28^{\circ} \mathrm{C}$ in $30 \mathrm{~mL}$ of Luria-Bertani (LB) medium containing rifampicin and kanamycin. Cells were grown to the stationary phase $\left(\mathrm{an} \mathrm{OD}_{600}\right.$ of approximately 2.0$)$ and harvested by centrifugation at $4500 \times g$ for $10 \mathrm{~min}$. The pellet was resuspended in medium $(0.5 \times$ MS, $5 \%$ sucrose, and $0.05 \%$ Silwett L-77) and DMSO or $100 \mu \mathrm{M}$ TNX was added to the Agrobacterium suspension. We performed drop-wise inoculation of individual flower buds using a micropipette. Seeds collected from inoculated plants were sown on medium supplemented with $50 \mathrm{mg} / \mathrm{L}$ kanamycin and $100 \mathrm{mg} / \mathrm{L}$ cefotaxime sodium and incubated for approximately 10 days to assess kanamycin resistance.

\section{Jatropha}

In this study, we transformed Jatropha (Jatropha curcas L.) using A. tumefaciens (strain EHA101) harbouring selection vectors (PalSelect A-3 vector, Kumiai Chemical Industry Co., Ltd.). The transformation procedure used has been described previously by Enoki et al. (2017). 
Briefly, 3-5-mm square segments of cotyledons (Fig. 2B) were co-cultivated with Agrobacterium suspension supplemented with DMSO or $100 \mu \mathrm{M}$ TNX at $26^{\circ} \mathrm{C}$ for 4 days in the dark. Calli and shoots that had developed after culturing on CIM followed by shoot induction medium, were transferred to selection medium. Elongated shoots were subsequently transferred to root induction medium and when roots had become fully developed, the transformed plants were transferred to soil and acclimatised in a growth room until genotyping by PCR using primers specific for the transgene.

292

\section{Maize}

294 For the transformation of maize (Zea mays L.), we used the inbred line Mi29 and A. tumefaciens (strain LBA4404) harbouring a pVGW9 helper plasmid carrying the bar gene driven by a ubiquitin promoter. The method used for transformation has been described previously by Ishida et al. (2007). Immature embryos obtained at approximately 10 to 18 days (depending on the season) after pollination, were co-cultivated on LSAS medium containing DMSO or $100 \mu \mathrm{M}$ $\mathrm{TNX}$ at $25^{\circ} \mathrm{C}$ for 7 days. The embryos were then transferred to LSD1.5A medium containing 3 $\mathrm{mg} / \mathrm{mL}$ bialaphos, $250 \mathrm{mg} / \mathrm{L}$ carbenicillin, and $100 \mathrm{mg} / \mathrm{L}$ cefotaxime and incubated for 10-20 days.

302

303

\section{Rice}

304 For rice (Oryza sativa L.) transformation, we used the cultivar Nipponbare and A. tumefaciens (strain EHA105, Hood et al., 1993) harbouring a monitoring vector (Saika et al., 2012). The transformation procedure followed that described by Toki (1997). Briefly, 1-week-old primary calli were co-cultivated with an Agrobacterium suspension supplemented with DMSO or $100 \mu \mathrm{M}$ TNX at $23^{\circ} \mathrm{C}$ for 3 days. Agrobacterium-infected calli were subsequently cultured on selection medium containing $10 \mathrm{mg} / \mathrm{L}$ blasticidin S (Nacalai Tesque) and $25 \mathrm{mg} / \mathrm{L}$ meropenem (Wako Pure Chemical Industries) at $31-33^{\circ} \mathrm{C}$. Transformed calli were treated with Beetle Luciferin, 311 potassium salt (Promega) and left to stand for $5 \mathrm{~min}$. Thereafter, Luc luminescence images were obtained and analysed using a Lumazone imaging system (Roper Scientific).

\section{Soybeans}

For the purpose of soybean [Glycine max (L.) Merr.] transformation, we used the Japanese soybean variety Kariyutaka, which was obtained from the Hokkaido Prefectural Agricultural Experiment Station, Tokachi, and is identical to the resource JP 86520 available from the Genebank Project, National Agriculture and Food Research Organization. Agrobacteriummediated transformation was performed using A. tumefaciens (strain EHA105) as previously described by Yamada et al. (2014). Either DMSO or $20 \mu \mathrm{M}$ TNX was added to the co-cultivation medium. Integration of the transgene was assessed by applying Basta (Bayer Crop Science) to the leaves of $\mathrm{T}_{1}$ seedlings.

\section{Brassica napus}

Brassica napus cv. Westar was transformed by inoculating hypocotyl sections with $A$. tumefaciens (strain GV3101) harbouring a binary vector containing the neomycin phosphotransferase II gene and regenerated as described by Kohno-Murase et al. (1994). DMSO or $100 \mu \mathrm{M}$ TNX was added to the co-cultivation medium. The numbers of transformed plants grown on selection medium were counted.

\section{Brassica rapa}


332 The seeds of Brassica rapa L. subsp. nipposinica ('Kyo-nishiki' cultivar of Mibuna) used for 333 transformation were purchased from Takii \& Co., Ltd., Kyoto, Japan. These seeds were sterilised 334 in 70\% ethanol for $2 \mathrm{~min}$, soaked and swirled in 1\% sodium hypochlorite for $15 \mathrm{~min}$, and then 335 washed five times with sterile distilled water to remove the sodium hypochlorite. The surface336 sterilised seeds were poured into a magenta box containing germination medium [1× MS 337 medium (Wako, Japan) containing $3 \%$ sucrose, $0.1 \%$ PPM (Plant Cell Technology, USA), and $3380.4 \%$ Gelrite (Wako, Japan)], and incubated at $20^{\circ} \mathrm{C}$ for 7 days for germination. Hypocotyl 339 explants from the germinated plants were subsequently pre-cultured for 3 days on CIM [B5 salt and vitamins (Wako), 1.5\% sucrose, 0.2\% PPM, and $1 \mathrm{mg} / \mathrm{L} 2-4 \mathrm{D}, \mathrm{pH} 5.8$ )]. A. tumefaciens (strain C58C1) carrying the binary vector pBCR101 was cultured overnight at $28^{\circ} \mathrm{C}$ in liquid $\mathrm{LB}$ medium containing $50 \mathrm{mg} / \mathrm{L}$ kanamycin. The following day, the Agrobacterium cells were harvested by centrifugation at 8,000 rpm for $20 \mathrm{~min}$ and then re-suspended to an $\mathrm{OD}_{600}$ of 0.1 with infection medium (Liquid MS, pH 5.8, and $200 \mu \mathrm{M}$ acetosyringone) containing DMSO or 100 $\mu \mathrm{M}$ TNX. The pre-cultured hypocotyl explants were soaked in the bacterial suspension for 10 min and then transferred to sterile paper to remove the Agrobacterium. The infected hypocotyl explants were then placed on CIM and incubated at $20^{\circ} \mathrm{C}$ for 3 days in the dark. Following cocultivation, the explants were washed with sterile distilled water and cultured on CIM containing $200 \mathrm{mg} / \mathrm{L}$ carbenicillin for 7 days, and subsequently transferred to fresh CIM at 2week intervals. After one and half months, calli had formed from the hypocotyl explants. As we were unable to obtain sufficient redifferentiation using the Mibuna cultivar, transformation efficiency was assessed by GUS staining of callus that developed from the hypocotyl explants.

\section{Water starwort}

Seeds of water starwort (Callitriche palustris L.), collected from a laboratory culture strain (originally collected from Nagano, Japan) were sterilised with $4 \%$ sodium hypochlorite solution (Wako, Japan) for $20 \mathrm{~min}$ and then incubated in sterile distilled water for 3-7 days to induce germination. The germinated seedlings were thereafter transplanted onto germination medium [0.5× MS medium (Wako, Japan) containing 2\% sucrose and $0.3 \%$ Gellan Gum (Wako, Japan)], and cultured in a growth chamber (NK system, Osaka, Japan) at $23^{\circ} \mathrm{C}$ under constant light at an illumination intensity of $60 \mu \mathrm{mol} \mathrm{m}{ }^{-2} \mathrm{~s}^{-1}$. Immediately prior to transfection, hypocotyls were dissected from 2-week-old seedlings as explants. A. tumefaciens (strain C58C1Rifr) harbouring a pH35G binary vector, in which a cDNA sequence for GFP was linked to the $35 S \mathrm{CaMV}$ promoter, were cultured in LB medium supplemented with $50 \mathrm{mg} / \mathrm{L}$ ampicillin and $100 \mathrm{mg} / \mathrm{L}$ spectinomycin at $28^{\circ} \mathrm{C}$ for $24 \mathrm{~h}$. For transfection, the Agrobacterium was resuspended in $0.5 \times$ MS supplemented with $2 \%$ sucrose. The explants were immersed in the Agrobacterium suspension containing D MSO or $50 \mu \mathrm{M}$ or $100 \mu \mathrm{M}$ TNX in addition to $100 \mu \mathrm{M}$ acetosyringone, and then incubated for $10 \mathrm{~min}$. The explants were thereafter transferred onto a solid medium consisting of $0.5 \times$ MS, $2 \%$ sucrose, $2 \mathrm{mg} / \mathrm{L}$ trans-zeatin (Tokyo Chemical Industry Co., LTD, Toyo, Japan), and $100 \mu \mathrm{M}$ acetosyringone. After co-cultivation for 3 days at $23^{\circ} \mathrm{C}$ in the dark, the Agrobacterium was removed by incubating the explants on $0.5 \times$ MS medium containing 250 $\mathrm{mg} / \mathrm{L}$ cefotaxime sodium salt (Sanofi K.K., Tokyo, Japan), 2\% sucrose, and $2 \mathrm{mg} / \mathrm{L}$ trans-zeatin for 2 days under illuminated growth conditions. The numbers of explants containing GFPpositive cells were counted under an MZ16 fluorescence stereomicroscope (Leica Microsystems, Wetzlar, Germany).

\section{List of abbreviations}




\section{Competing interests}

The authors declare that they have no competing interests

\section{Author contributions}

S-WC, KS and YI conceived and designed the experiments. KK, RM, HE, WC, TT, HS, ME, TY, AH, NK, SK, YK, and HK performed the experiments. YI wrote the first draft of the manuscript. S-WC, $\mathrm{EI}, \mathrm{KS}$, and YI reviewed and edited the manuscript. All authors contributed to the article and approved the submitted version.

\section{Funding}

This research was supported by a NARO grant-in-aid (20902 to HS), a Promotion of Science Grant in Aid for JSPS Fellow (14J08122 to HK), Building of Consortia for the Development of Human Research in Science and Technology, MEXT, Japan (to EI), KAKENHI (17H06172 to KS), and PRESTO, Japan Science and Technology Agency (JPMJPR15Q2 to YI).

\section{Acknowledgements}

We thank Dr. Shuta Asai (Riken Center for Sustainable Resource Science) for providing seeds of the dde2/ein2/pad4/sid2 quadruple mutant, Dr. Hirokazu Tsukaya (The University of Tokyo) for providing helpful advice regarding the experiments using water starwort, and Dr. Goro Horiguchi (Rikkyo University) for providing the binary vector pH35G.

\section{References}

Altpeter, F., Springer, N. M., Bartley, L. E., Blechl, A. E., Brutnell, T. P., Citovsky, V., et al. (2016). Advancing crop transformation in the era of genome editing. Plant Cell 28, 1510-1520. doi:10.1105/tpc. 16.00196.

Anand, A., Uppalapati, S. R., Ryu, C.-M., Allen, S. N., Kang, L., Tang, Y., et al. (2008). Salicylic acid and systemic acquired resistance play a role in attenuating crown gall disease caused by Agrobacterium tumefaciens. Plant Physiol. 146, 703-715. doi:10.1104/pp.107.111302.

Chandran, D., Rickert, J., Huang, Y., Steinwand, M. A., Marr, S. K., and Wildermuth, M. C. (2014). Atypical E2F transcriptional repressor DEL1 acts at the intersection of plant growth and immunity by controlling the hormone salicylic acid. Cell Host Microbe 15, 506-513. doi:10.1016/j.chom.2014.03.007.

Choi, S., Tamaki, T., Ebine, K., Uemura, T., Ueda, T., and Nakano, A. (2013). RABA members act in distinct steps of subcellular trafficking of the FLAGELLIN SENSING2 receptor. Plant Cell 25, 1174-1187. doi:10.1105/tpc.112.108803.

de Groot, M. J. A., Bundock, P., Hooykaas, P. J. J., and Beijersbergen, A. G. M. (1998). Agrobacterium tumefaciens-mediated transformation of filamentous fungi. Nat. Biotechnol. 16, 839-842. doi:10.1038/nbt0998-839.

Enoki, H., Funato, A., Nabetani, Y., Takahashi, S., Ichikawa, T., Matsui, M., et al. (2017). Chapter 12. Using an improved method of Agrobacterium-mediated transformation to attempt to make larger seeds to increase oil production. The Jatropha Genome, 191-203.

Escobar, M. A., and Dandekar, A. M. (2003). Agrobacterium tumefaciens as an agent of disease. Trends Plant Sci. 8, 380-386. doi:10.1016/S1360-1385(03)00162-6.

Forson, F. K., Oduro, E. K., and Hammond-Donkoh, E. (2004). Performance of jatropha oil blends in a diesel engine. Renew. Energy 29, 1135-1145. doi:https://doi.org/10.1016/j.renene.2003.11.002.

Gaspar, Y. M., Nam, J., Schultz, C. J., Lee, L.-Y., Gilson, P. R., Gelvin, S. B., et al. (2004). Characterization of the Arabidopsis lysine-rich arabinogalactan-protein AtAGP17 mutant (rat1) that results in a decreased efficiency of agrobacterium transformation. Plant Physiol. 135, 2162-2171. doi:10.1104/pp.104.045542. 
Gelvin, S. B. (2005). Agricultural biotechnology: gene exchange by design. Nature 433, 583-584. doi:10.1038/433583a.

Goff, S. A., Ricke, D., Lan, T.-H., Presting, G., Wang, R., Dunn, M., et al. (2002). A draft sequence of the rice genome (Oryza sativa L. ssp japonica). Science 296, 92-100. doi:10.1126/science.1068275.

Hiei, Y., Ohta, S., Komari, T., and Kumashiro, T. (1994). Efficient transformation of rice (Oryza sativa L.) mediated by Agrobacterium and sequence analysis of the boundaries of the TDNA. Plant J. 6, 271-282. doi:10.1046/j.1365-313x.1994.6020271.x.

Hood, E. E., Gelvin, S. B., Melchers, L. S., and Hoekema, A. (1993). NewAgrobacterium helper plasmids for gene transfer to plants. Transgenic Res. 2, 208-218. doi:10.1007/BF01977351.

Ishida, Y., Hiei, Y., and Komari, T. (2007). Agrobacterium-mediated transformation of maize. Nat. Protoc. 2, 1614-1621. doi:10.1038/nprot.2007.241.

Ishida, Y., Saito, H., Ohta, S., Hiei, Y., Komari, T., and Kumashiro, T. (1996). High efficiency transformation of maize (Zea mays L.) mediated by Agrobacterium tumefaciens. Nat. Biotechnol. 14, 745-750. doi:10.1038/nbt0696-745.

Ishihama, N., Choi, S., Noutoshi, Y., Saska, I., Asai, S., Takizawa, K., et al. (2020). Oxicam-type nonsteroidal anti-inflammatory drugs inhibit NPR1-mediated salicylic acid pathway. bioRxiv, 2020.09.25.311100. doi:10.1101/2020.09.25.311100.

Kadota, Y., Watanabe, T., Fujii, S., Higashi, K., Sano, T., Nagata, T., et al. (2004). Crosstalk between elicitor-induced cell death and cell cycle regulation in tobacco BY-2 cells. Plant J. 40, 131142. doi:10.1111/j.1365-313X.2004.02197.X.

Kimura, M., Cutler, S., and Isobe, S. (2015). A novel phenolic compound, chloroxynil, improves agrobacterium-mediated transient transformation in Lotus japonicus. PLoS One 10, e0131626. doi:10.1371/journal.pone.0131626.

Kohno-Murase, J., Murase, M., Ichikawa, H., and Imamura, J. (1994). Effects of an antisense napin gene on seed storage compounds in transgenic Brassica napus seeds. Plant Mol. Biol. 26, 1115-1124. doi:10.1007/BF00040693.

Komari, T., Takakura, Y., Ueki, J., Kato, N., Ishida, Y., and Hiei, Y. (2006). Binary vectors and super-binary vectors. Methods Mol. Biol. 343, 15-41. doi:10.1385/1-59745-130-4:15.

Kunik, T., Tzfira, T., Kapulnik, Y., Gafni, Y., Dingwall, C., and Citovsky, V. (2001). Genetic transformation of HeLa cells by Agrobacterium. Proc. Natl. Acad. Sci. U. S. A. 98, 1871-1876. doi:10.1073/pnas.98.4.1871.

Lee, C.-W., Efetova, M., Engelmann, J. C., Kramell, R., Wasternack, C., Ludwig-Müller, J., et al. (2009). Agrobacterium tumefaciens promotes tumor induction by modulating pathogen defense in Arabidopsis thaliana. Plant Cell 21, 2948-2962. doi:10.1105/tpc.108.064576.

Nonaka, S., Sugawara, M., Minamisawa, K., Yuhashi, K.-I., and Ezura, H. (2008a). 1Aminocyclopropane-1-carboxylate deaminase enhances Agrobacterium tumefaciensmediated gene transfer into plant cells. Appl. Environ. Microbiol. 74, 2526-2528. doi:10.1128/AEM.02253-07.

Nonaka, S., Yuhashi, K.-I., Takada, K., Sugaware, M., Minamisawa, K., and Ezura, H. (2008b). Ethylene production in plants during transformation suppresses vir gene expression in Agrobacterium tumefaciens. New Phytol. 178, 647-656. doi:10.1111/j.14698137.2008.02400.x.

Noutoshi, Y., Okazaki, M., Kida, T., Nishina, Y., Morishita, Y., Ogawa, T., et al. (2012). Novel plant immune-priming compounds identified via high-throughput chemical screening target salicylic acid glucosyltransferases in Arabidopsis. Plant Cell 24, 3795-3804. doi:10.1105/tpc.112.098343.

Piers, K. L., Heath, J. D., Liang, X., Stephens, K. M., and Nester, E. W. (1996). Agrobacterium tumefaciens-mediated transformation of yeast. Proc. Natl. Acad. Sci. U. S. A. 93, 1613-1618. doi:10.1073/pnas.93.4.1613.

Que, Q., Elumalai, S., Li, X., Zhong, H., Nalapalli, S., Schweiner, M., et al. (2014). Maize transformation technology development for commercial event generation. Front. Plant Sci. 5, 379. doi:10.3389/fpls.2014.00379. 
482

483

484

485

486

487

488

489

490

491

492

493

494

495

496

497

498

499

500

501

502

503

504

505

506

507

508

509

510

511

512

513

514

515
Rashid, H., Yokoi, S., Toriyama, K., and Hinata, K. (1996). Transgenic plant production mediated by Agrobacterium in Indica rice. Plant Cell Rep. 15, 727-730. doi:10.1007/BF00232216.

Rohini, V. K., and Rao, S. (2000). Transformation of peanut (Arachis hypogaea L.): A non-tissue culture based approach for generating transgenic plants. Plant Sci. 150, 41-49. doi:10.1016/S0168-9452(99)00160-0.

Rosas-Díaz, T., Cana-Quijada, P., Amorim-Silva, V., Botella, M. A., Lozano-Durán, R., and Bejarano, E. R. (2017). Arabidopsis NahG plants as a suitable and efficient system for transient expression using Agrobacterium tumefaciens. Mol. Plant 10, 353-356. doi:10.1016/j.molp.2016.11.005.

Saika, H., Nonaka, S., Osakabe, K., and Toki, S. (2012). Sequential monitoring of transgene expression following Agrobacterium-mediated transformation of rice. Plant Cell Physiol. 53, 1974-1983. doi:10.1093/pcp/pcs135.

Sardesai, N., Lee, L.-Y., Chen, H., Yi, H., Olbricht, G. R., Stirnberg, A., et al. (2013). Cytokinins secreted by Agrobacterium promote transformation by repressing a plant myb transcription factor. Sci. Signal. 6, ra100. doi:10.1126/scisignal.2004518.

The Arabidopsis Genome Initiative (2000). Analysis of the genome sequence of the flowering plant Arabidopsis thaliana. Nature 408, 796-815. doi:10.1038/35048692.

Tsuda, K., Sato, M., Stoddard, T., Glazebrook, J., and Katagiri, F. (2009). Network properties of robust immunity in plants. PLoS Genet. 5, e1000772. doi:10.1371/journal.pgen.1000772.

Tzfira, T., Vaidya, M., and Citovsky, V. (2004). Involvement of targeted proteolysis in plant genetic transformation by Agrobacterium. Nature 431, 87-92. doi:10.1038/nature02857.

Wang, W., and Wang, Z.-Y. (2014). At the intersection of plant growth and immunity. Cell Host Microbe 15, 400-402. doi:10.1016/j.chom.2014.03.014.

Wenck, A. R., Quinn, M., Whetten, R. W., Pullman, G., and Sederoff, R. (1999). High-efficiency Agrobacterium-mediated transformation of Norway spruce (Picea abies) and loblolly pine (Pinus taeda). Plant Mol. Biol. 39, 407-416. doi:10.1023/A:1006126609534.

Yu, J., Hu, S., Wang, J., Wong, G. K.-S., Li, S., Liu, B., et al. (2002). A draft sequence of the rice genome (Oryza sativa L. ssp. indica). Science 296, 79-92. doi:10.1126/science.1068037.

Yuan, Z.-C., Edlind, M. P., Liu, P., Saenkham, P., Banta, L. M., Wise, A. A., et al. (2007). The plant signal salicylic acid shuts down expression of the vir regulon and activates quormonequenching genes in Agrobacterium. Proc. Natl. Acad. Sci. U. S. A. 104, 11790-11795. doi:10.1073/pnas.0704866104. 
516 Figure legends

517 Figure 1. The structures of oxicam compounds. Tenoxicam (TNX), meloxicam (MLX),

518 piroxicam (PRX), ampiroxicam (APRX), sudoxicam (SDX), and lornoxicam (LNX).

519

Figure 2. Oxicam treatment enhances the efficiency of Agrobacterium-mediated transient transformation in Arabidopsis thaliana

523

(A and B) Leaves of wild-type and dde2/ein2/pad4/sid2 quadruple mutant Arabidopsis thaliana

524 were mock- and tenoxicam [TNX $(100 \mu \mathrm{M})]$-treated and inoculated with Agrobacterium carrying

525 a 35S::GUS. (A) After 2 days, GUS enzyme activity was determined by histochemical staining and

526 a quantitative MUG assay. The blue colour indicates GUS activity. Experiments were repeated

527 five times with similar results. Bars represent the means and standard errors of data obtained from three biological replicates. (B) Transgenic leaves inoculated with Agrobacterium strains carrying the constructs RPS::H2B-GFP for the nucleus, 35S::Venus-SYP61 for the trans-Golgi network, and 35S::PIP2A-mCherry for the plasma membrane in the mock- or TNX $(100 \mu \mathrm{M})$ treated wild-type plants. Fluorescence signals in epidermal cells were observed 2 days after infiltration. Bars $=100 \mu \mathrm{m}$. (C and D) Quantitative MUG assay to assess the effect of TNX on quadruple mutant plants (C) and the effect of oxicams on wild-type plants (D). Agroinfiltration conditions were the same as those in (A). Different letters indicate statistically significant

Figure 3. Tenoxicam (TNX) treatment enhances the efficiency of Agrobacterium-mediated stable transformation in Jatropha

539

(A and B) Images of mature Jatropha plants (A) and the cotyledon used for transformation (B).

540 Bar, $50 \mathrm{~mm}$. (C) Transformation rate determined by PCR genotyping in the mock- or TNX (100 $\mu \mathrm{M}$ )-treated plants. The data obtained from two independent experiments are shown. Numbers above the bars indicate number of transformants with respect to the number of treated soilacclimated plants.

Figure 4. Tenoxicam (TNX) treatment enhances the efficiency of stable Agrobacteriummediated transformation in maize

548 (A) Image of the immature embryo of maize that was used for transformation. Bar, $1 \mathrm{~mm}$. (B) Transformation rate determined based on survival rate on selection medium for mock- and TNX $(100 \mu \mathrm{M})$-treated plants. The data obtained from six independent experiments using different treatments. Bar $=1 \mathrm{~cm}$. 


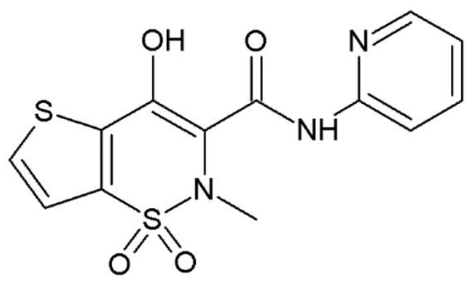

Tenoxicam (TNX)

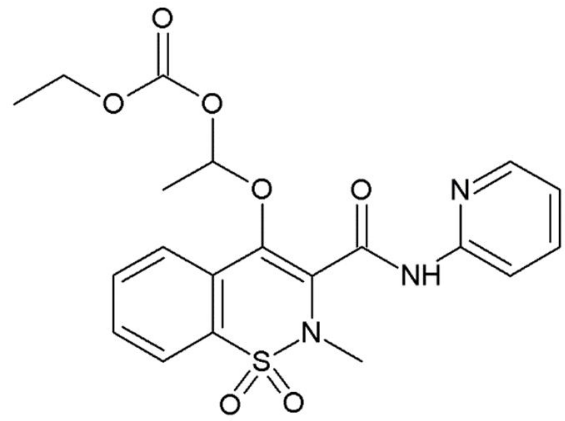

Ampiroxicam (APRX)<smiles>Cc1cnc(NC(=O)C2=C(O)c3ccccc3S(=O)(=O)N2C)s1</smiles>

Meloxicam (MLX)

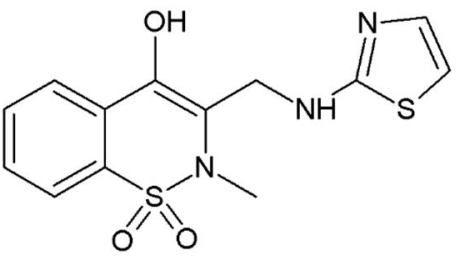

Sudoxicam (SDX)<smiles>CN1C(C(=O)Nc2ccccn2)=C(O)c2ccccc2S1(=O)=O</smiles>

Piroxicam (PRX)<smiles>CN1C(C(=O)Nc2ccccn2)=C(O)c2sc(Cl)cc2S1(=O)=O</smiles>

Lornoxicam (LNX) 
(A)

(B)

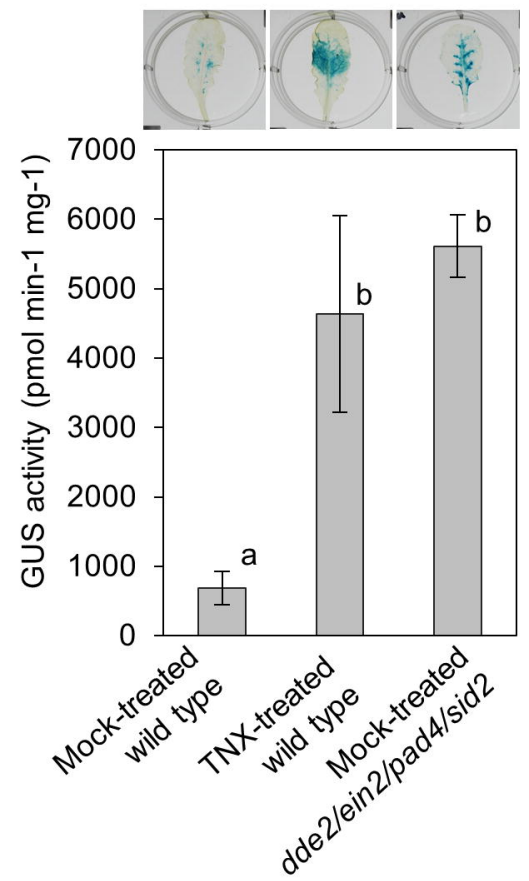

(C)

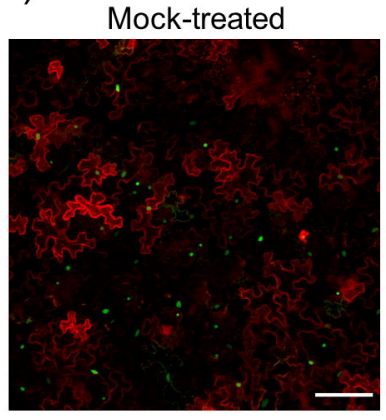

TNX-treated

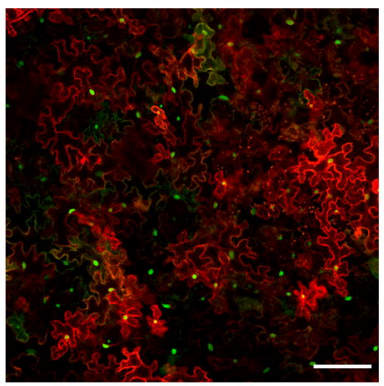

(D)
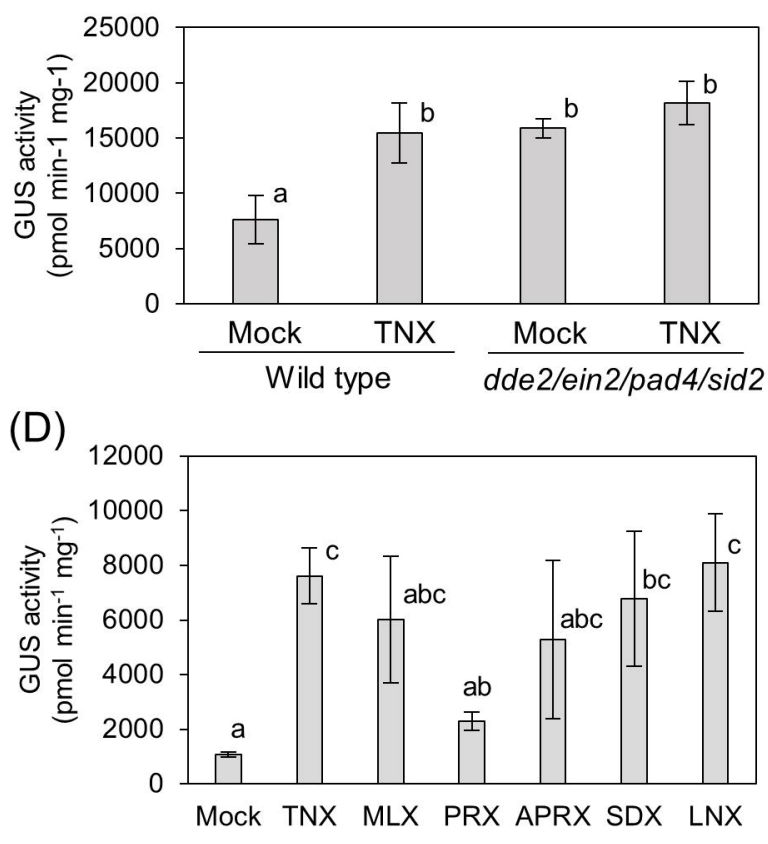


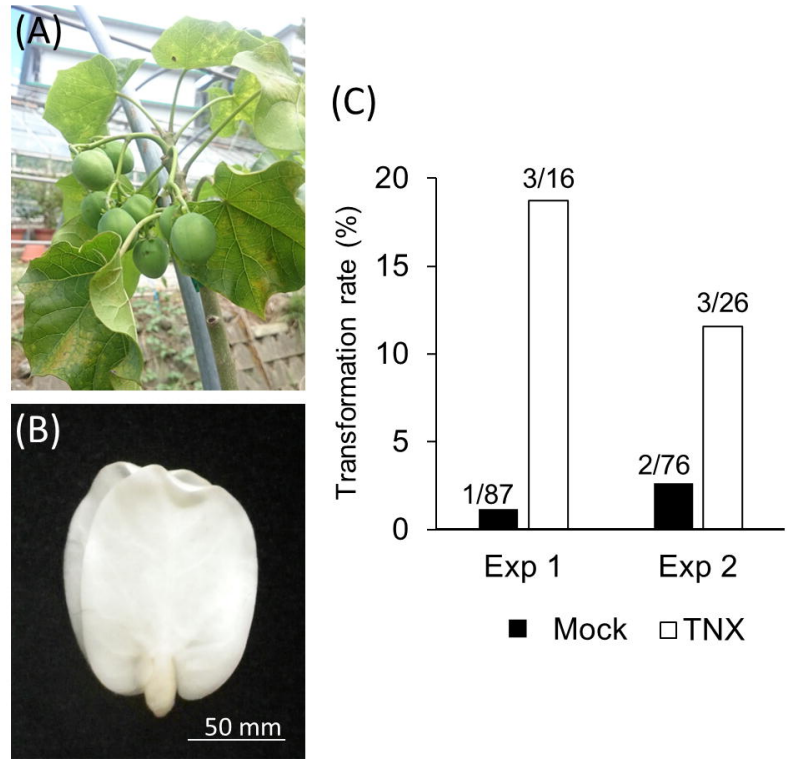


